\title{
Identification professionnelle ou suridentification à la profession?
}

\author{
La situation de directrices et de directeurs d'étalblissements \\ scolaires québécois \\ Professional Identification or Over-Identification with the \\ Profession and Québec School Principals \\ ¿Identificación profesional o sobre-identificación a la \\ profesión? \\ La situación de las directoras y los directores de \\ establecimientos escolares quebequences
}

\section{Lise Corriveau}

Volume 32, numéro 2, automne 2004

Administrateur, administratrice scolaire et identité professionnelle

URI : https://id.erudit.org/iderudit/1079074ar

DOI : https://doi.org/10.7202/1079074ar

Aller au sommaire du numéro

Éditeur(s)

Association canadienne d'éducation de langue française

ISSN

0849-1089 (imprimé)

1916-8659 (numérique)

Découvrir la revue

Citer cet article

Corriveau, L. (2004). Identification professionnelle ou suridentification à la profession? La situation de directrices et de directeurs d'établissements scolaires québécois. Éducation et francophonie, 32(2), 95-110. https://doi.org/10.7202/1079074ar
Résumé de l'article

Cet article présente les résultats d'une recherche (Brassard Brassard, Corriveau, Fortin, Gélinas, Savoie-Zajc, 2001), réalisée au moyen d’un questionnaire et d'entrevues semi-structurées auprès des directrices et des directeurs d'établissements scolaires francophones des ordres primaire et secondaire du Québec, pour connaitre leurs opinions sur les divers changements mis en oeuvre à la suite de l'implantation de la réforme scolaire québécoise qui a débuté durant l'année 1997-1998. Les résultats de cette recherche indiquent clairement que la tâche des directrices et des directeurs d'établissements s'est alourdie et complexifiée. Malgré le fait qu'ils consacrent plus de temps et d'énergie à leur travail, la plupart des directrices et des directeurs rencontrés en entrevue se disent néanmoins satisfaits et toujours aussi passionnés par leur travail. Dans ces conditions, cet article tente de répondre aux questions suivantes : Quel est l'impact de la réforme scolaire sur la tâche des directrices et des directeurs d'établissements? Combien de temps consacrent-ils à leur travail? Y a-t-il danger, pour les directrices et les directeurs d'établissements scolaires de glisser subtilement vers une suridentification à la profession? Comment les directrices et les directeurs parviennent-ils à établir l'équilibre entre leur vie personnelle et professionnelle? En ce sens, les résultats de cette recherche révèlent que la plupart des directrices et des directeurs interviewés sont conscients du danger de glisser vers la suridentification professionnelle et des risques qu'ils encourent pour leur santé physique et mentale. Cependant, plusieurs font part des divers moyens individuels qu'ils utilisent pour éviter de tomber en déséquilibre et suggèrent des moyens organisationnels que pourraient prendre les commissions scolaires pour les aider à conserver leur bien-être ou à rétablir leur équilibre pour une identification saine à leur profession.

Tous droits réservés ( $\odot$ Association canadienne d'éducation de langue française, Ce document est protégé par la loi sur le droit d’auteur. L’utilisation des 2004 services d'Érudit (y compris la reproduction) est assujettie à sa politique d'utilisation que vous pouvez consulter en ligne.

https://apropos.erudit.org/fr/usagers/politique-dutilisation/ 


\section{Identification professionnelle ou suridentification à la profession? \\ La situation de directrices et de directeurs d'établissements scolaires québécois}

\section{Lise Corriveau}

Départements de la Gestion de l'éducation et de la formation, Université de Sherbrooke, Sherbrooke, (Québec) Canada

\section{RÉSUMÉ}

Cet article présente les résultats d'une recherche (Brassard Brassard, Corriveau, Fortin, Gélinas, Savoie-Zajc, 2001), réalisée au moyen d'un questionnaire et d'entrevues semi-structurées auprès des directrices et des directeurs d'établissements scolaires francophones des ordres primaire et secondaire du Québec, pour connaître leurs opinions sur les divers changements mis en œuvre à la suite de l'implantation de la réforme scolaire québécoise qui a débuté durant l'année 1997-1998. Les résultats de cette recherche indiquent clairement que la tâche des directrices et des directeurs d'établissements s'est alourdie et complexifiée. Malgré le fait qu'ils consacrent plus de temps et d'énergie à leur travail, la plupart des directrices et des directeurs rencontrés en entrevue se disent néanmoins satisfaits et toujours aussi passionnés par leur travail.

Dans ces conditions, cet article tente de répondre aux questions suivantes : Quel est l'impact de la réforme scolaire sur la tâche des directrices et des directeurs d'établissements? Combien de temps consacrent-ils à leur travail? Y a-t-il danger, pour les 
directrices et les directeurs d'établissements scolaires de glisser subtilement vers une suridentification à la profession? Comment les directrices et les directeurs parviennent-ils à établir l'équilibre entre leur vie personnelle et professionnelle? En ce sens, les résultats de cette recherche révèlent que la plupart des directrices et des directeurs interviewés sont conscients du danger de glisser vers la suridentification professionnelle et des risques qu'ils encourent pour leur santé physique et mentale. Cependant, plusieurs font part des divers moyens individuels qu'ils utilisent pour éviter de tomber en déséquilibre et suggèrent des moyens organisationnels que pourraient prendre les commissions scolaires pour les aider à conserver leur bien-être ou à rétablir leur équilibre pour une identification saine à leur profession.

\section{ABSTRACT}

\section{Professional Identification or Over-Identification with the Profession and Québec School Principals}

Lise Corriveau, Departments of Education Management and Training, University of Sherbrooke, Sherbrooke, (Québec) Canada

This article presents the results of a research project (Brassard Brassard, Corriveau, Fortin, Gélinas, Savoie-Zajc, 2001) based on a questionnaire and loosely structured interviews with principals of francophone schools at both the primary and secondary levels, gathering their opinions on various changes resulting from the implantation of the Québec school reform begun in the 1997-1998 school year. The results of this research clearly show that the duties of school principals have become heavier and more complex. Despite the fact that they spend more time and energy doing their work, most principals interviewed say that they are nevertheless satisfied and still passionate about their work.

Under these conditions, this article tries to answer the following questions: What is the impact of the school reform on the school principal's job? How much time to they devote to their work? Is there a danger that principals may subtly slide towards over-identifying with the profession? How can principals establish a balance between their personal and professional lives? In this sense, the results of this research revealed that most of the principals interviewed are conscious of the danger of professional over-identification and the risks to their physical and mental health. However, several share a variety of individual methods they use to avoid falling into disequilibrium, and suggest organizational means school boards could take to help them maintain their well-being, or re-establish the equilibrium necessary for healthy identification with the profession. 


\section{RESUMEN}

\section{¿Identificación profesional o sobre-identificación a la profesión? La situación de las directoras y los directores de establecimientos escolares quebequences.}

Lise Corriveau, Departamentos de la gestión de la educación y de la formación, Universidad de Sherbrooke, Sherbrooke, (Quebec) Canadá

Este artículo presenta los resultados de una investigación ((Brassard Brassard, Corriveau, Fortin, Gélinas, Savoie-Zajc, 2001), realizada mediante un cuestionario y entrevistas semi-estructuradas entre las directoras y directores de establecimientos escolares francófonos de niveles primario y secundario de Quebec, con el fin de conocer sus opiniones sobre los diversos cambios introducidos por la reforma escolar quebequence, que se inició en el año de 1997-1998. Los resultados de esta investigación indican claramente que la tarea de las directoras y directores de los establecimientos ha aumentado y se ha vuelto más compleja. A pesar de que dedican más tiempo y energía a su trabajo, la mayor parte de los directores y directoras entrevistadas se sienten satisfechos y están contentos con su trabajo.

En esas condiciones, este artículo trata de responder a las cuestiones siguientes: ¿Cuál ha sido el impacto de la reforma escolar sobre la carga de trabajo de las directoras y los directores de los establecimientos? ¿Cuánto tiempo consagran a su trabajo? ¿Existe el peligro, para las directoras y los directores de los establecimientos escolares de caer en una sobre-identificación con la profesión? ¿Cómo logran los directores y las directoras establecer el equilibrio entre sus vidas personales y su vida profesional? Al respecto, los resultados de esta investigación rebelan que la mayor parte de directoras y directores entrevistados están conscientes del peligro de caer en una sobre-identificación profesional así como de los riesgos que corre su salud física y mental. Sin embargo, algunos hablan de los medios individuales que utilizan para evitar de caer en el desequilibrio y sugieren medios organizacionales que podrían adoptar las comisiones escolares para ayudarlos a conservar su bienestar o para restablecer su equilibrio y una identificación sana con su profesión. 


\section{Introduction}

À la suite de la mise en application de la Loi sur l'Instruction publique (1998), des résultats de recherche ${ }^{1}$ (Brassard, Corriveau, Fortin, Gélinas, Savoie-Zajc, 2001) indiquent clairement que la tâche des directrices et des directeurs d'établissements s'est alourdie et complexifiée. Engagés dans la mise en œuvre de la réforme scolaire,

La tâche des directrices et des directeurs d'établissements s'est alourdie et complexifiée. passionnés et dévoués à leur travail, animés par les nombreux défis qui façonnent leur quotidien, les directrices et les directeurs consacrent une bonne partie de leur temps à l'accomplissement de leur travail. Même si la majorité considère leur travail très stimulant, il n'en demeure pas moins que la tâche est exigeante physiquement, émotionnellement et intellectuellement et qu'elle empiète souvent sur la vie privée (Corriveau, 2002). Dans ces conditions, y a-t-il danger, pour les directrices et les directeurs d'établissements scolaires de glisser subtilement vers une suridentification professionnelle? Quels problèmes peut poser trop d'identification avec le travail? Comment les directrices et les directeurs parviennent-ils à établir l'équilibre entre leur vie personnelle et professionnelle? Le but de cet article est d'apporter un éclairage à ces questions à partir des résultats de notre recherche (Brassard, Corriveau, Fortin, Gélinas, Savoie-Zajc, 2001).

Cet article se divise trois en parties. La première présente le contexte et le cadre de référence permettant de situer la problématique. La deuxième partie décrit la méthodologie utilisée dans notre recherche. La troisième présentent les résultats, leur analyse et leur interprétation tout en offrant une liste de moyens individuels et organisationnels susceptibles d'aider les directrices et les directeurs à maintenir ou à atteindre une vie plus équilibrée.

\section{Contexte}

À l'instar d'autres pays européens et américains, le ministère de l'Éducation du Québec a entrepris, durant l'année 1997-1998, une réforme axée sur une décentralisation administrative et une transformation du curriculum qui demande aux établissements scolaires de fonctionner différemment. Sur le plan administratif, il institue une décentralisation des pouvoirs scolaires des commissions scolaires vers les établissements et met en place les conseils d'établissement pour orienter le développement des écoles en fonction des besoins particuliers des milieux. Sur le plan pédagogique, la réforme du curriculum met en vigueur un nouveau programme de formation qui vise le développement des compétences et une organisation scolaire par cycle qui incitent fortement les intervenants à modifier leurs pratiques pédagogiques.

1. Sondage et entrevues réalisés par le GRIDES (Groupe de recherche interuniversitaire sur les directions d'établissement scolaire, André Brassard, Lise Corriveau, Régent Fortin, Arthur Gélinas, Lorraine Savoie-Zajc) Recherche subventionnée par le Conseil de recherche en sciences humaines du Canada (CRSH, 1998-2001). 
Les premiers interpellés par ces transformations sont les gestionnaires scolaires, particulièrement les membres de la direction des établissements, car le rôle de piloter ces changements leur revient.

En 1986, à la suite d'une étude d'observation réalisée auprès de quatre directeurs d'école secondaire, Pépin (1986, dans Brassard et al., 1986) indiquait que le travail des directrices et des directeurs d'école était marqué par un volume élevé de travail, un rythme accéléré, la brièveté, la variété et la fragmentation des tâches. Ainsi, les directeurs observés travaillaient en moyenne 48 heures par semaine et effectuaient en moyenne 171 tâches distinctes par jour; $89 \%$ de leurs activités duraient moins de 5 minutes et seulement $5 \%$, plus de 10 minutes; enfin, $66 \%$ de toutes les activités étaient interrompues ou interrompaient d'autres activités en cours. À la suite d'une réforme scolaire mise en œuvre à Chicago en 1988 (Bennett et al., 1992), une étude collaborative réalisée par questionnaire auprès de 450 directrices et directeurs d'établissements primaires et secondaires pour connaître leurs attitudes envers la réforme, révèle une augmentation de la quantité de travail à accomplir et du temps à consacrer à l'exercice de leur fonction. Ainsi, les répondantes et les répondants disent consacrer en moyenne 60 heures par semaine à leur travail et passer plus de temps que nécessaire à des tâches administratives au détriment du

Imputables de l'amélioration de la réussite de leurs élèves dans leur école, ils disent manquer de temps et de ressources pour y arriver. temps passé sur des aspects pédagogiques. Par ailleurs, ils considèrent que la réforme leur demande le déploiement d'un plus grand nombre de compétences et qu'ils ont peu de ressources pour développer le leadership nécessaire à la mise en œuvre de la réforme. Même s'ils sont très dévoués à leur travail, ils trouvent leur tâche trop lourde pour le temps dont ils disposent. Imputables de l'amélioration de la réussite de leurs élèves dans leur école, ils disent manquer de temps et de ressources pour y arriver. Dans cette recherche, une question est clairement posée: combien de temps les directrices et les directeurs d'établissements scolaires pourront-ils maintenir un tel rythme de travail avec les contraintes qu'ils connaissent?

Ces résultats rejoignent ceux de l'enquête sur la relève réalisée par Charuest (2001) à la demande de la Fédération des Commissions scolaires du Québec. Cette enquête révèle les facteurs qui incitent des enseignantes et des enseignants ( $n=159)$ à ne pas poser leur candidature à un poste de direction d'école. Parmi les cinq premiers facteurs énoncés, nous retrouvons, par ordre d'importance, la lourdeur de la tâche, les longues heures de travail, les rencontres et les réunions le soir ou les fins de semaine, l'empiètement de la tâche et des activités sur la vie personnelle et le cumul de deux demi-tâches. Tous ces facteurs ont trait à la charge de travail et à l'équilibre entre la vie personnelle et la vie professionnelle. Il s'agit vraisemblablement d'un refus de la part de ces enseignantes et de ces enseignants de se surinvestir au travail. Selon Laferrière (2002), la lourdeur de la tâche contribue actuellement au Québec à la pénurie de directrices et de directeurs d'école dont la moitié des postes devront être comblés d'ici cinq ans.

Au moment de la mise en application de la réforme scolaire au Québec, nous tenterons, dans cet article, de répondre aux questions suivantes : Quel est l'impact de la réforme scolaire sur la tâche des directrices et des directeurs d'établissements? Combien de temps consacrent-ils à leur travail? Y a-t-il danger, pour les directrices et 
les directeurs d'établissements scolaires de glisser subtilement vers une suridentification à la profession? Comment les directrices et les directeurs parviennent-ils à établir l'équilibre entre leur vie personnelle et professionnelle? Le but de cet article est d'apporter un éclairage à ces questions à partir des résultats obtenus à un sondage et aux entrevues réalisées auprès de directrices et de directeurs d'établissement scolaires répartis à travers la province de Québec. ${ }^{2}$

\section{Identification ou suridentification professionnelle}

Plusieurs caractéristiques distinctes contribuent à former l'identité d'une personne et l'ensemble de ces caractéristiques détermine ce qu'elle est. Inspirée de Gohier et al. (1999, p. 29), nous pouvons définir l'identité professionnelle comme l'image qu'une personne se fait de son travail, de ses responsabilités, de ses rapports aux autres ainsi que de son appartenance au groupe et à l'organisation comme institution sociale. Le développement de cette identité repose à la fois sur des processus d'identification (similitudes avec les autres qui exercent la même profession ou identification à la profession en soi) et d'identisation (processus où la personne se reconnaît comme étant «elle-même» dans l'exercice de sa profession, avec sa personnalité et ses valeurs.)

Lorsque l'identification à la profession prend beaucoup de place dans le développement de l'identité, on peut alors parler de «suridentification professionnelle ». Il y a suridentification professionnelle lorsque le travail devient l'élément central de la vie d'un individu, lorsque ce dernier devient complètement absorbé par son travail au détriment des autres secteurs de sa vie ou des autres rôles qu'il est appelé à jouer. Pour certaines personnes, la carrière devient «l'élément organisateur de la vie personnelle et le principe qui lui confère un sens. " (Aubert, dans Pauchant, 1996, p. 106) En conséquence, pour Fontana (1990), l'importance ou l'estime que se porte une personne devient alors dépendante et indissociable de son travail.

Les écrits portant directement sur la suridentification professionnelle sont peu nombreux. Pour décrire cette réalité, des concepts tels "dépendance existentielle", « comportement de type A» et «boulotmanie» sont plus souvent utilisés.

Le concept de dépendance existentielle, tel qu'utilisé par Bracke et Bugental (dans Pauchant, 1996, p. 78) s'apparente à celui de la suridentification professionnelle: «une façon d'être dans le monde dans lequel une activité, habituellement le travail, devient progressivement le centre de la vie. La dépendance supplante peu à peu tous les autres aspects de la vie.» Même si on y retrouve des éléments semblables à la suridentification professionnelle telle que l'entend Fontana (1990), Bracke et Bugental (ibid.) soutiennent toutefois que la dépendance existentielle prend son origine non pas d'une déficience sur le plan de l'estime de soi, mais plutôt d'un sentiment d'insécurité personnelle ou d'un désir d'éviter la détresse liée à l'anxiété et au

2. Sondage et entrevues réalisés par le GRIDES (Groupe de recherche interuniversitaire sur les directions d'établissement scolaire, André Brassard, Lise Corriveau, Régent Fortin, Arthur Gélinas, Lorraine Savoie-Zajc) recherche subventionnée par le CRSH (1998-2001). 
vide. Les personnes affectées de dépendance existentielle s'immergent et s'engourdissent dans une activité, souvent le travail, pour éviter de faire face à une anxiété existentielle, comme « le changement, la mort et la contingence, la responsabilité, la renonciation, le deuil et l'isolement. » (Bruke et Bugental, dans Pauchant, 1996, p. 79)

Les comportements de type A et la «boulotmanie" peuvent être considérés comme des manifestations de la dépendance existentielle. Toutefois, ils peuvent aussi être apparentés à la suridentification professionnelle puisqu'il est possible qu'ils soient engendrés par une déficience sur le plan de l'estime de soi. Indépendamment de son origine, on peut définir ainsi le comportement de type A :

Le comportement de type A est caractérisé par « une lutte permanente non seulement contre le temps (impatience, rapidité dans l'action, plusieurs activités menées simultanément), mais aussi contre les autres (compétitivité importante, ambition sociale élevée, etc.) et par des états émotionnels hostiles fréquents en situation sociale (colère, agressivité exprimée ou contenue, etc.).» (Légeron, 2003, p. 219)

En complément, Brake et Bugental (dans Pauchant, 1996, p. 77), ajoutent que les sujets de type A présentent une «dépendance à la productivité, à la perfection et au contrôle». Pour leur part, les «boulotmanes" partagent des caractéristiques fort semblables à celles du profil de type A.

Il est important d'établir une distinction entre les comportements de type A, la «boulotmanie », la dépendance existentielle et l'engagement, le dévouement et le réel intérêt qu'une personne porte à son travail. Pour plusieurs personnes, le travail permet de relever des défis intéressants et constitue une source importante de satisfaction et d'épanouissement dans une vie équilibrée. (Brake et Bugental, dans Pauchant, 1996). Par ailleurs, il n'y a pas nécessairement de mal à se "suridentifier » à un seul secteur de sa vie si cette image correspond à celle que l'on souhaite et qu'on valorise. Toutefois, les comportements associés à la suridentification professionnelle comportent des risques qu'il importe de considérer.

Un premier risque est lié au sentiment de vide personnel (Bruke et Bugental, dans Pauchant, 1996) qui peut survenir lorsqu'une personne laisse de côté ses besoins et ses intérêts personnels. L'excès dans la vie professionnelle peut entraîner une négligence de la vie personnelle qui alors se détériore et amène l'individu à réinvestir dans sa vie professionnelle. Cela s'inscrit dans une boucle sans fin.

Un deuxième risque est lié non seulement à l'équilibre de la personne, mais aussi au bien-être de ses proches. Ainsi, le surinvestissement d'un individu au travail peut se faire au détriment de ses besoins personnels, mais aussi de ses autres rôles (parent, conjoint, ami, etc.) et le conduire à un désengagement progressif envers sa vie familiale, sociale et collective (ACSQ, 2000).

Enfin, comme dans le cas précis de dépendance existentielle, certaines personnes trouveront dans le travail un refuge leur permettant de fuir les insatisfactions liées à leur vie personnelle. Tant que leur travail sera gratifiant, ces personnes pourront y trouver les satisfactions nécessaires à leur équilibre. Toutefois, un troisième risque lié à la suridentification apparaît lorsque le travail cesse d'être satisfaisant. 
Selon Aubert (dans Pauchant, 1996, p. 117),

Cette perte de reconnaissance est particulièrement difficile à vivre dans les cas où s'était opéré, entre l'individu et l'entreprise, un collage trop fort, une symbiose trop grande, lorsque le moi de l'individu avait fini par fusionner en quelque sorte avec l'idéal de l'organisation. On assiste alors à ces phénomènes de dépression brutale où la personne «craque», parfois soudainement, parfois en plusieurs étapes.

La suridentification au travail peut alors mener au développement des maladies liées au stress ou à l'épuisement professionnel caractérisé par une grande fatigue sur les plans physique, émotif et intellectuel (Gareau, 2003).

Une vie personnelle riche permettant de développer diverses facettes de sa per-

La suridentification au travail peut alors mener au développement des maladies liées au stress ou à l'épuisement professionnel. sonnalité de même que le soutien des êtres chers peuvent aider à relativiser la place donnée au travail, à traverser les moments plus difficiles dans sa carrière et à maintenir un équilibre physique, émotionnel et intellectuel. Il y aurait donc avantage à maintenir une gestion équilibrée des différentes facettes de sa vie. Avec la mise en place de la réforme scolaire, quelle place prend le travail dans la vie des directrices et des directeurs d'établissements scolaires? Parviennent-ils à maintenir un équilibre entre leur vie personnelle et professionnelle? Comment y arrivent-ils?

\section{Les aspects méthodologiques}

Le Groupe de Recherche Interuniversitaire sur les Directions d'Établissement Scolaire (GRIDES) a conduit une recherche auprès des directrices et des directeurs d'établissements scolaires francophones des ordres primaires et secondaires d'enseignement du Québec pour connaître leurs opinions sur les divers changements en éducation au Québec et la gestion des établissements scolaires depuis la mise en œuvre de la réforme (Brassard et al., 2001). La méthodologie utilisée dans cette recherche est mixte. Elle utilise les méthodes de cueillette de données quantitatives et qualitatives. Cette recherche se veut descriptive et s'inscrit dans le paradigme compréhensif et interprétatif.

Les données de cette recherche ont été recueillies en deux phases. À l'hiver 2000, un sondage par questionnaire a été réalisé par le GRIDES auprès des 3100 directrices et directeurs d'établissements scolaires francophones des ordres primaires et secondaires d'enseignement du Québec ${ }^{3}$. Au total, 544 questionnaires ont été retournés pour un taux de réponse de $17,5 \%$. De ce nombre, $80,6 \%$ occupaient un poste de direction et $19,4 \%$ un poste d'adjoint à la direction. Lors de cette étape, les répondantes et les répondants étaient invités à nous indiquer sous pli séparé s'ils acceptaient de participer à la deuxième phase de notre recherche en nous recevant en entrevue. Plus de 250 directeurs ont donné une réponse positive.

3. Avec la collaboration et le soutien de I'AMDES, de I'AQPDE et de la FQDE que nous remercions. 
À la fin de l'année 2000 et au début de l'année 2001, les cinq chercheurs du GRIDES ont donc procédé à une deuxième cueillette d'informations qui s'est déroulée sous la forme d'entrevues semi-structurées effectuées auprès de 68 directeurs d'établissements œuvrant dans les différentes régions du Québec. Il faut spécifier qu'à cette période, la réforme du curriculum était en vigueur depuis quatre à six mois pour le premier cycle du primaire (depuis septembre 2000), soit pour les classes de maternelle, de première et de deuxième année. À peu près la moitié des 68 entrevues ont été effectuées auprès des personnes nous ayant signifié leur intérêt à poursuivre leur participation à la recherche. L'autre moitié des personnes ont été sélectionnées en dehors de celles qui s'étaient portées volontaires. L'âge et le sexe des répondants, leurs années d'expérience dans la fonction de direction, l'ordre d'enseignement de l'établissement, la taille de celui-ci, la commission scolaire d'appartenance, la région et toutes autres caractéristiques particulières propres à la situation de la directrice ou du directeur ou de son établissement (par exemple, le fait pour une directrice ou un directeur de diriger deux écoles) ont été considérés afin de constituer un échantillon diversifié de répondantes et de répondants. Une fois transcrites, les entrevues on fait l'objet d'un codage détaillé à l'aide du logiciel N'Vivo.

\section{Présentation des résultats}

Cette section présente dans un premier temps les résultats obtenus au questionnaire (première phase). Les résultats obtenus aux entrevues (deuxième phase) seront ensuite exposés; ils permettront de comprendre et d'interpréter les résultats recueillis dans la première phase de notre recherche.

\section{Analyse des résultats au sondage}

Parmi tous les résultats obtenus à la suite de notre enquête par questionnaire, seuls ceux reflétant la perception des directrices et des directeurs concernant les effets de la Loi sur l'Instruction publique sur les caractéristiques reliées à l'exercice

Les résultats de notre recherche indiquent clairement que la tâche des directrices et des directeurs d'établissement s'est alourdie et complexifiée. de la fonction de direction seront ici retenus. Ainsi, à la suite de la mise en application de la Loi sur l'Instruction publique (1998), les résultats de notre recherche indiquent clairement que la tâche des directrices et des directeurs d'établissement s'est alourdie et complexifiée. Les résultats présentés au tableau 1 sont éloquents : la majorité des 544 répondants de notre sondage (2001) perçoivent que le temps consacré à la fonction, le nombre d'activités à accomplir, la vitesse d'exécution, la complexité de leur tâche et le stress lié à la fonction a augmenté 4 . En ce qui a trait au temps destiné plus précisément à la tâche, les répondants estiment que c'est le temps consacré aux mécanismes de prise de décision et de consultation dans l'établissement et celui consacré aux aspects administratifs qui ont largement augmenté. En contrepartie, la portion du temps consacré aux aspects pédagogiques semble avoir diminué. Ainsi, 43,2 \% des répondants considèrent passer moins de temps aux aspects pédagogiques alors que $37,3 \%$ disent en consacrer plus. Pour $19,5 \%$ des

4. Il faut lire «a augmenté » ou « a beaucoup augmenté ». 
répondants, le temps consacré est resté semblable. Enfin, 29,4\% des répondants s'estiment moins satisfaits dans l'exercice de leur fonction, contre $30,6 \%$ qui se disent plus satisfaits. Pour les autres (40\%), la satisfaction éprouvée dans l'exercice de la fonction est restée semblable.

Tableau 1. Les effets de la Loi sur l'Instruction publique sur les caractéristiques reliées à l'exercice de la fonction de direction

\begin{tabular}{|l|c|c|c|}
\cline { 2 - 4 } \multicolumn{1}{c|}{} & A diminué & Est semblable & A augmenté \\
\hline Le temps consacré à I'exercice de la fonction & $(14)$ & $(54)$ & $(450)$ \\
\hline Le nombre d'activités à accomplir & $2,7 \%$ & $10,5 \%$ & $86,8 \%$ \\
\hline La vitesse d'exécution & $(2)$ & $(19)$ & $(504)$ \\
& $0,4 \%$ & $3,6 \%$ & $96 \%$ \\
\hline La complexité dans l'exercice de la fonction & $(6)$ & $(68)$ & $(448)$ \\
\hline Le stress de la fonction & $1,2 \%$ & $13,0 \%$ & $85,8 \%$ \\
\hline Le temps consacré aux mécanismes de prise de & $(2)$ & $(36)$ & $(488)$ \\
décision et de consultation dans I'établissement & $0,4 \%$ & $6,8 \%$ & $92,8 \%$ \\
\hline Le temps consacré aux aspects administratifs & $(3)$ & $(92)$ & $(429)$ \\
\hline Le temps consacré aux aspects pédagogiques & $(11)$ & $17,6 \%$ & $81,9 \%$ \\
\hline La satisfaction éprouvée dans I'exercice de & $(10)$ & $(59)$ & $(455)$ \\
\hline la fonction & $(152)$ & $11,3 \%$ & $86,6 \%$ \\
\hline
\end{tabular}

Ces résultats rejoignent ceux de l'enquête réalisée à la suite de la réforme scolaire de la région de Chicago et de celle réalisée au Québec sur la relève par Charuest (2001). On observe ainsi qu'à la suite de la réforme, les directrices et les directeurs ont le sentiment d'effectuer une tâche plus lourde, plus complexe, qui exige de plus longues heures de travail et estiment passer plus de temps aux aspects administratifs de la tâche. Déjà, en 1986, Pépin estimait que la tâche effectuée par les directrices et les directeurs d'établissement était considérable. Que signifient les résultats présentés au tableau 1 dans la vie quotidienne des directrices et des directeurs d'établissement scolaire québécois?

\section{Analyse des résultats aux entrevues semi-structurées}

Comme il a été mentionné précédemment, 68 entrevues semi-structurées ont été réalisées auprès de directrices et de directeurs d'établissements d'ordres primaire et secondaire à travers le Québec. Il n'y avait pas de question permettant aux direc- 
trices et aux directeurs de se situer directement quant au phénomène de la suridentification professionnelle. Toutefois, quatre questions ont conduit des répondantes et des répondants à s'exprimer sur le sujet :

- Est-ce que les changements modifient quelque chose dans l'exercice de votre fonction?

- Comment vous sentez-vous personnellement et professionnellement à travers tous ces changements?

- Est-ce que dans tout cela, vous pensez gagner quelque chose ou perdre quelque chose?

- Est-ce que vous êtes plus satisfaits ou moins satisfaits qu'avant dans votre tâche?

Au total, 30 directrices et directeurs d'établissements des ordres d'enseignement primaire et secondaire ont fourni des données utiles à notre analyse sur la suridentification. Les résultats seront analysés selon une logique inductive modérée, en fonction des dimensions suivantes, à savoir : l'impact de la réforme scolaire sur la tâche, l'impact de la réforme sur le temps qui y est consacré, l'impact de l'augmentation de la tâche sur soi, l'équilibre entre la vie personnelle et la vie professionnelle, les dangers qui guettent les directrices et les directeurs d'établissement scolaire, et des pistes pour atteindre une vie plus équilibrée.

Le tableau 2 présente la distribution des 30 répondantes et répondants en fonction des années d'expérience, de leur fonction et de leur sexe.

Tableau 2. Distribution des répondantes et des répondants en fonction des années d'expérience, de leur fonction et de leur sexe

\begin{tabular}{|l|c|c|c|}
\cline { 2 - 4 } \multicolumn{1}{c|}{} & \multicolumn{3}{c|}{ Années d'expérience } \\
\cline { 2 - 4 } \multicolumn{1}{c|}{} & $\begin{array}{c}\text { Moins de } \mathbf{5} \text { ans } \\
\text { f'expérience }\end{array}$ & $\begin{array}{c}\text { Entre } \mathbf{5} \text { et } \mathbf{1 0} \text { ans } \\
\mathbf{d} \text { d'expérience }\end{array}$ & $\begin{array}{c}\text { Plus de } \mathbf{1 0} \text { ans } \\
\text { d'expérience }\end{array}$ \\
\hline Directrice primaire & 3 & 3 & 8 \\
\hline Directeur primaire & 1 & 1 & 4 \\
\hline Directrice secondaire & & 1 & 2 \\
\hline Directeur secondaire & & 2 & \\
\hline Directeur primaire/secondaire & & 1 & \\
\hline Adjointe secondaire & 1 & & \\
\hline Adjoint secondaire & 1 & & \\
\hline
\end{tabular}

\section{Impact de la réforme scolaire sur la tâche}

Avec la mise en place de la réforme scolaire, les directrices et les directeurs de notre échantillon s'entendent pour dénoncer la lourdeur et la complexité de leur tâche, telle que révélée par les résultats au questionnaire. Les demandes provenant du ministère de l'Éducation et des commissions scolaires (par exemple, les plans de 
réussite, la reddition de comptes) dans des échéanciers serrés, l'ajout de nouveaux dossiers liés à la décentralisation des pouvoirs dans les écoles (budget, comptabilité), les coupures de personnel dans certains cas (secrétariat, direction adjointe), les nouvelles structures de décentralisation (conseil d'établissement, travail en équipe, gestion participative, rôle politique, préparation des dossiers, suivi des dossiers), l'absence ou la diminution de ressources accompagnant la décentralisation dans d'autres et la multiplication des informations fournies par l'efficacité des nouvelles technologies comptent parmi les éléments qui contribuent à nourrir ce sentiment de lourdeur administrative et qui ajoutent à la complexité de la tâche tant chez les directrices et les directeurs du primaire que ceux du secondaire, débutants ou expérimentés. Un directeur d'un établissement secondaire décrit, en ces mots, la lourdeur de la tâche : «La fonction de leader pédagogique à l'école devrait prendre $75 \%$, puis la fonction administrative $25 \%$, et bien, il faut calculer que la fonction administrative prend $85 \%$ du temps, ça fait que le $75 \%$, il faut que tu l'ajoutes, ça fait que tu montes à $150 \%$.» Ces résultats suivent de près ceux récoltés en 1992 à la suite de la réforme scolaire de la région de Chicago. Charge de travail plus lourde, manque de ressources, tâche administrative dominante, ces facteurs confirment aussi ceux révélés par l'enquête de Charuest (2001) et qui concourent à la pénurie des directrices et des directeurs d'établissements (Charuest, 2001; Laferrière, 2002).

\section{Temps consacré au travail}

La lourdeur de la tâche ajoute définitivement des heures à la semaine de travail des directrices et des directeurs d'établissements scolaires. D'une moyenne de 48 heures consacrées par semaine au travail en 1986 (Pépin, 1986), on observe une augmentation d'au moins 10 heures de travail par semaine en 2001. Ainsi, plusieurs parmi les directrices et les directeurs ont indiqué travailler en moyenne 60 heures et même jusqu'à 70 heures par semaine. Les journées débutent autour de 7 h30 pour se terminer entre 18 et 19 heures. Certains affirment prendre très peu de temps pour dîner. Le travail se poursuit parfois en soirée en raison des réunions (conseil d'établissement, rencontres avec des parents, etc.). D'autres disent apporter du travail à la maison la fin de semaine ou «se taper régulièrement un samedi ou un dimanche à son école». Est-il possible d'organiser sa tâche de travail autrement? Une directrice du primaire nous dit : «On a beau avoir un sens de l'organisation et de la délégation, ça a des limites, ça aussi. Il y a des choses que je ne peux pas faire autrement... ". Ces résultats abondent dans le sens de ceux recueillis par l'étude de Chicago (1992). Cependant, deux directrices apportent des réponses différentes. L'une d'entre elles, moyennement expérimentée, exprime clairement son refus de travailler le midi, sauf exceptionnellement: "Il faut se faire violence pour ne pas faire ce qu'ils font, travailler le midi, parce qu'on ne peut pas se voir ailleurs, avec un dossier à côté de notre soupe ». Même si certains considèrent leur tâche difficilement conciliable avec le fait d'avoir de jeunes enfants, une jeune directrice du primaire, mère de famille, indique terminer ses réunions avant le souper: «Moi, je suis une jeune mère de famille, j'ai des enfants d'âge scolaire. Moi, à 5h30, mes réunions sont finies. Il n'y en a pas sur 
l'heure du souper. " Ainsi, même si le travail prend beaucoup de place, les résultats indiquent déjà des moyens que certains utilisent pour maintenir l'équilibre dans leur vie.

\section{Impact sur soi}

Les résultats au questionnaire indiquent que $60 \%$ des directrices et directeurs éprouvent une satisfaction semblable ou même plus grande dans l'exercice de leur fonction depuis la réforme, alors que $29,4 \%$ d'entre eux s'estiment moins satisfaits. Lors des entrevues, il s'avère intéressant de constater des résultats qui vont dans la même direction. Ainsi, malgré la lourdeur et la complexité de la tâche qui les accaparent, la majorité des directrices et des directeurs de notre échantillon ressentent pour la plupart un bien-être vis-à-vis des changements qui ont cours actuellement dans le système d'éducation. Ils disent se sentir "très bien", «à l'aise ", «très confortable", devant les changements qui surviennent et les défis qu'ils soulèvent, même s'ils deviennent fatigués à un moment donné. Ils aiment être au cœur de l'action, ils aiment "quand ça bouge, quand ça évolue, quand il y a des défis». Bref, ils adorent ce qu'ils font même si cela s'avère très exigeant sur les plans intellectuel, physique et émotionnel. Même en début de carrière, un directeur adjoint au secondaire nous dit: «Avec la réforme qui s'en vient, moi ça m’enchante. Même si je sais qu'il va y avoir des embûches, j'aime ça... Je me dis, quand on réussit, on a une satisfaction.» Quelques-uns affirmeront cependant vivre des sentiments d'insatisfaction devant la multitude et la rapidité des changements qui les empêchent de faire les choses « correctement» ou encore de faire les choses «qui apporteraient encore plus aux jeunes qu'on a entre les mains ».

En somme, même s'ils consacrent un nombre considérable d'heures à leur travail et qu'ils subissent une fatigue certaine, les directrices et les directeurs rencontrés sont, pour la plupart, satisfaits et toujours passionnés par leur travail. On retrouve ici les caractéristiques des personnes pour lesquelles le travail permet de relever des défis intéressants et qui, malgré la charge importante de travail, y trouvent une

Les directrices et les directeurs interviewés semblent conscients de la très grande place que prend leur travail au détriment des autres sphères de leur vie. importante source de satisfaction et d'épanouissement. Dans ces cas, on pourrait parler d'un engagement et d'un dévouement sains au travail (Brake et Bugental, dans Pauchant, 1996). Toutefois, la fatigue est présente; certains indiquent même une fatigue importante face à la tâche. Quels sont les risques encourus? Comment préserver l'équilibre entre la vie personnelle et la vie professionnelle?

\section{Équilibre entre la vie personnelle et la vie professionnelle}

Les directrices et les directeurs interviewés semblent conscients de la très grande place que prend leur travail au détriment des autres sphères de leur vie. Aussi, la plupart témoigne d'une préoccupation certaine pour ne pas laisser envahir complètement leur vie personnelle. L'investissement qu'ils font dans leur vie intime, dans les relations personnelles et familiales, dans leurs loisirs, la volonté qu'ils ont de se respecter, de se donner des moments juste pour eux, les aident grandement à départager la vie personnelle de la vie professionnelle, à rétablir leur équilibre et à se donner une qualité de vie. Quelques-unes, parce qu'elles ont des enfants plus âgés ou un conjoint compréhensif, y trouvent l'occasion de s'investir encore plus dans 
leur vie professionnelle. Toutefois, même si la tâche est exigeante en temps et en énergie, la plupart directrices et les directeurs ont pris conscience du besoin de protéger leur équilibre et leur bien-être.

\section{Les dangers qui guettent les directrices et les directeurs d'établissement scolaire}

Des dangers liés à une suridentification au travail ont été mentionnés par les directrices et les directeurs d'établissements, dangers qu'ils ont identifiés à partir de leur propre expérience ou de celles de leurs collègues. Leur grande passion pour leur travail, jumelée à la lourdeur et à la complexité de la tâche, à l'absence ou au manque de ressources, au désir que certains ont de plaire à tous malgré l'application de décisions impopulaires, au sentiment de ne pas être toujours à la hauteur des performances exigées et parfois, au manque de reconnaissance de la commission scolaire, des pairs ou des collaborateurs, contribuent principalement, selon eux, au développement du stress, aux diverses maladies qui lui sont reliées et à l'épuisement professionnel (Gareau, 2003). "Quand autour de toi, tu as des confrères, des consœurs que tu admires, qui ont le même âge que toi puis qui, tout à coup meurent, ou qui se retrouvent avec des maladies graves... Je dirais que si j'avais à perdre quelque chose, ça serait la santé».

\section{Pistes pour atteindre une vie plus équilibrée}

La plupart des directrices et les directeurs d'établissements rencontrés disent utiliser ou mettre en place des moyens personnels pour modérer les effets du stress engendré par leur travail. Ils évoquent également des moyens organisationnels que pourraient prendre les commissions scolaires pour les aider à rétablir leur équilibre tant sur le plan physique, émotif qu'intellectuel. La liste est longue mais parmi ceux qui ont été mentionnés, notons :

\section{Moyens individuels}

\section{Vie personnelle}

- Avoir la volonté ferme de s'occuper de soi, de protéger sa vie personnelle

- Définir ses propres limites et ses priorités

- Se donner suffisamment d'heures de sommeil

- Se mettre en forme physiquement

- $\quad$ Prendre du temps compensatoire pour les réunions du soir

- Partager du temps avec son conjoint et ses enfants, mêmes grands

\section{Vie professionnelle}

- Participer à des groupes d'entraide, de support

- Départager les problèmes qui leur appartiennent de ceux qui appartiennent aux autres 
- Ne pas régler les problèmes des autres mais les accompagner dans la recherche de leurs propres solutions

- $\quad$ Prendre du recul, de la distance, par rapport aux événements stressants

- Se centrer sur les besoins des élèves

- Se donner les moyens d'être à l'aise avec le changement et les nouvelles technologies

\section{Moyens organisationnels}

- $\quad$ Réduire les tâches administratives

- Ajouter du personnel, des services, des ressources

- Offrir des sessions de formation sur le temps de travail pour ne pas allonger la semaine de travail

- Recevoir appui, support et reconnaissance de la commission scolaire

\section{Conclusion}

À la suite de la réforme scolaire, les résultats recueillis par le GRIDES indiquent clairement que la fonction de direction d'établissement scolaire est plus lourde et plus complexe qu'auparavant. Parmi les 68 directeurs et directrices interviewées, 30 nous ont confié, dans leurs mots, leurs inquiétudes quant aux dangers de glisser vers la suridentification professionnelle et des risques qu'ils encourent pour leur santé physique et mentale. Fait étonnant, ce constat ne semble pas se limiter aux directrices et aux directeurs qui débutent dans la fonction, mais s'étendre également chez les gestionnaires plus expérimentés. On ne peut généraliser ce constat à l'ensemble des directrices et des directeurs qui ne se sont pas prononcés sur le sujet. Cependant, d'autres études (ACSQ, 2002; Bennett et al., 1992; Charuest, 2001) indiquent, à ce jour, des résultats similaires.

Tous aussi passionnés les uns que les autres pour leur travail, ils investissent le meilleur d'eux-mêmes quotidiennement sans compter les heures pour répondre aux besoins des élèves qui leur sont confiés, et ce, dans un environnement souvent marqué par les coupures de personnel, de services ou de ressources. Face à ces résultats, il est heureux de constater que plusieurs disent utiliser divers moyens pour éviter de tomber en déséquilibre. En somme, il est du ressort de chaque individu d'agir en faisant des choix, en modifiant ses attitudes et en établissant des priorités dans sa vie personnelle et professionnelle. Les répondantes et les répondants ont aussi mentionné le souhait de voir les commissions scolaires mettre en œuvre des moyens organisationnels pour les aider à conserver leur bien-être ou à rétablir leur équilibre : réduire les tâches administratives, ajouter, dans la mesure du possible, du personnel, des services, des ressources, leur permettre de suivre les formations sur le temps de travail pour éviter d'allonger les semaines en soirée et les fins de semaine, les soutenir, les appuyer et les reconnaître dans leur travail. Il est primordial que les organisations apportent leur soutien aux personnes qu'elles emploient pour favoriser leur bien-être de manière à ce qu'elles soient plus disponibles pour les autres et plus efficaces dans la prise de décisions et ce, au profit de la réussite des élèves. 
Identification professionnelle ou suridentification à la fonction? L'heure de la relève a sonné, mais celle-ci se fait rare. À court terme, des moyens concrets tant individuels qu'organisationnels devront être pris pour faire les choses autrement en vue d'améliorer la qualité de vie des gestionnaires d'établissements scolaires et d'intéresser à nouveau des enseignantes et des enseignants à s'identifier à cette profession qui soulève encore, et malgré tout, bien des passions!

\section{Références bibliographiques}

Alain, Marcel (1993). Prendre en main le changement. Stratégies personnelles et organisationnelles. Montréal : Éditions Nouvelles.

Association des cadres scolaires du Québec (Mars 2000). S'éduquer à la santé : le défi des gestionnaires. Bulletin de l'Association des cadres scolaires du Québec.

Aubert, N. (1996). L'entreprise comme instance de création existentielle: aspirations et désillusions, dans Pauchant, T. La quête du sens. Montréal : Presses HEC, pp. 101-120.

Brake, P.E., Bugental, J.F.T. (1996). La dépendance existentielle : traiter le comportement de type A et la boulotmanie, dans Pauchant, T. La quête du sens. Montréal: Presses HEC, pp. 73-100.

Brassard, A., Brunet, L., Corriveau, L., Pépin, R. et Martineau, G. (1986). Les rôles des directions d'école au Québec : $2^{e ̀ m e}$ partie. L'exercice des rôles et l'efficacité organisationnelle. Montréal : Université de Montréal, Faculté des sciences de l'éducation.

Brassard, A., Corriveau, L., Fortin, R., Gélinas, A., Savoie-Zajc, L., Héon, L. De Saedeleer, S. (2001). Les directions d'établissement et les changements en éducation. Groupe de recherche interuniversitaire sur les directions d'établissement scolaire (GRIDES).

Corriveau, L. (2002). Le gestionnaire en équilibre, une école en santé. Revue Le Point en administration scolaire. Vol. 4, No 4, pp. 16-17.

Fontana, D. (1990). Gérer le stress. Bruxelles : Pierre Mardaga, éditeur.

Gareau, A. (2003). Les gens épanouis... réussissent mieux. Outremont: Québécor.

Gohier, C., Anadon, M., Bouchard, Y., Charbonneau, B., Chevrier, J. (2001). Vers une vision renouvelée de la professionnalisation de l'enseignement et de la construction de l'identité professionnelle de l'enseignant. Dans C. Gohier, N. Bednarz, L. Gaudreau, R. Pallascio, G. Parent (dir.). L'enseignant un professionnel. Sainte-Foy: Presses de l'Université du Québec, pp. 21-56.

Laferrière, M. (2002). Pénurie de directeurs d'école. La Presse, 19 novembre 2002. p. A7. Légeron, P. (2003). Le stress au travail Paris : Odile Jacob. 